\title{
Two common polymorphic variants of OATP4A1 as potential risk factors for colorectal cancer
}

\author{
VERONIKA BUXHOFER-AUSCH ${ }^{1,2}$, ORSOLYA NÉMET ${ }^{3}$, MAJDAH SHEIKH $^{4}$, HAJNALKA ANDRIKOVICS ${ }^{5}$, \\ ANGELIKA REINER $^{6}$, CHRISTOPH AUSCH ${ }^{7}$, DIANA MECHTCHERIAKOVA ${ }^{4}$, ATTILA TORDAI ${ }^{8}$, \\ ANDREAS GLEISS $^{9}$, CSILLA ÖZVEGY-LACZKA ${ }^{3}$, WALTER JÄGER ${ }^{10}$ and THERESIA THALHAMMER ${ }^{4}$ \\ ${ }^{1}$ Department of Internal Medicine I with Hematology, Stem Cell Transplantation, Hemostaseology and Medical Oncology, \\ Ordensklinikum Linz der Elisabethinen, A-4020 Linz; ${ }^{2}$ Medical Faculty, Johannes Kepler University Linz, A-4040 Linz, \\ Austria; ${ }^{3}$ Membrane Protein Research Group, Institute of Enzymology, Research Centre for Natural Sciences, \\ H-1117 Budapest, Hungary; ${ }^{4}$ Institute of Pathophysiology and Allergy Research, Center for Pathophysiology, Infectiology and \\ Immunology, Medical University of Vienna, A-1090 Vienna, Austria; ${ }^{5}$ Laboratory of Molecular Genetics, \\ National Institute of Hematology and Infectious Diseases, Central Hospital of Southern Pest, H-1097 Budapest, Hungary; \\ ${ }^{6}$ Department of Pathology, Donauspital/Sozialmedizinisches Zentrum Ost, A-1220 Vienna; ${ }^{7}$ Department of Surgery, \\ Krankenhaus Göttlicher Heiland, A-1170 Vienna, Austria; ${ }^{8}$ Institute of Pathophysiology, Semmelweis University, \\ Faculty of Medicine, H-1085 Budapest, Hungary; ${ }^{9}$ Center for Medical Statistics, Informatics, and Intelligent Systems, \\ Medical University of Vienna; ${ }^{10}$ Department of Pharmaceutical Chemistry, University of Vienna, A-1090 Vienna, Austria
}

Received January 24, 2020; Accepted August 3, 2020

DOI: 10.3892/ol.2020.12115

\begin{abstract}
Genetic variations in the organic-anion-transporting polypeptide (OATP)-encoding solute carrier of organic anions (SLCO) genes can promote cancer development and progression. The overexpression of solute carrier organic anion transporter family member 4A1 (OATP4A1), a transporter for steroid hormones, prostaglandins, and bile acids, has been previously associated with tumor recurrence and progression in colorectal cancer (CRC). Therefore, the present study aimed to investigate the association between 2 frequent single nucleotide polymorphisms (SNPs) in SLCO4AI (rs34419428, R70Q; rs1047099G, V78I) and CRC predisposition. Following restriction fragment length polymorphism-PCR analysis in 178 patients with CRC [Union for International Cancer Control (UICC) stage I/II] and 65 healthy controls, no significant difference was observed in allele frequency and the number of heterozygous/homozygous individuals between the groups. Notably, the R70Q minor allele was identified
\end{abstract}

Correspondence to: Dr Theresia Thalhammer, Institute of Pathophysiology and Allergy Research, Center for Pathophysiology, Infectiology and Immunology, Medical University of Vienna, Waehringer Guertel 18-20/3Q, A-1090 Vienna, Austria

E-mail: theresia.thalhammer@meduniwien.ac.at

Key words: polymorphic variants, solute carrier organic anion transporter family member 4A1, solute carrier, colorectal cancer, colorectal cancer risk, single-nucleotide polymorphism, rs34419428, rs1047099 to be associated with the V78I minor allele in the genome. Comparing of the individual genotypes of CRC patients to clinical data, including sex, UICC-stage and relapse revealed no increased risk for CRC. In addition, the OATP4A1 immunoreactivity assay in paraffin-embedded CRC and adjacent non-tumorous mucosa sections, examined using quantitative microscopy image analysis, did not reveal any association with these polymorphisms. No significant differences were observed in the expression levels, localization, and sodium fluorescein transport capacity among the OATP4A1 variants, which was studied using functional assays in Sf9-insect and A431 tumor cells overexpressing the 2 single and a double mutant OATP4A1 SNP variants. These results suggested that the 2 most frequent polymorphisms located in the first intracellular loop of OATP4A1 do not associate with CRC predisposition and tumor recurrence. They are unlikely to affect the outcome of CRC in patients.

\section{Introduction}

Colorectal cancer (CRC) is one of the most prevalent types of cancer worldwide. Despite the existence of an established screening system, CRC remains to be the second leading cause of cancer-associated mortality in a number of countries. Therefore, the identification of novel biomarkers with therapeutic potentials is a subject of intense research (1). Persistent accumulation of genetic and epigenetic changes in the epithelial cells of the colon and rectum has been documented to promote the malignant transformation of normal mucosa into invasive cancer. Among the possible candidates of proteins associated with CRC are transporters belonging to the solute carrier (SLC) family. It was previously reported that solute carrier organic 
anion transporter family member 4A1 (OATP4A1), a transporter from the family of OATPs, encoded by the solute carrier organic anion $(S L C O) 4 A 1$ gene is overexpressed in CRC tumors $(2,3)$. Studies in cancer cell lines revealed further that OATP4A1 mediates the uptake of endogenous compounds including prostaglandin E2 (PGE2), sulfated and glucuronidated steroid hormone precursors, bile acids, and drugs such as benzylpenicillin and isoprostane (4-8). For the analysis of OATP-associated transport in vitro, OATP4A1-mediated uptake can be measured using the sodium fluorescein surrogate substrate (9). Notably, several endogenous OATP4A1 substrates have been identified to mediate a significant role in CRC by regulating signaling pathways that were implicated in the malignant transformation of the mucosa and tumor progression. The proinflammatory prostaglandin PGE2 is reportedly taken up from the extracellular space into the mucosa cells by OATP4A1, which facilitates its enzymatic inactivation to suppress its pro-inflammatory effects (10). In addition, bile acids are also substrates of OATP4A1, which are taken up by the mucosa cells for further metabolism to prevent their accumulation in the colonic lumen. This observation has been hypothesized to be beneficial as certain bile acids can promote carcinogenesis in the colon (11). OATP4A1 can also transport steroid hormone precursors such as estrone sulfate, which has been demonstrated to increase cell proliferation following its metabolization to $17 \beta$-estradiol, the most biologically active estrogen. Knockdown of OATP4A1 expression in colon cancer cells lines has been identified to significantly disrupt estrone sulfate uptake (12). However, the role of estrogen in CRC remains controversial. Previous epidemiological studies have suggested that estrogen-based hormone replacement therapy may decrease the risk of CRC, whilst data from in vitro studies demonstrated that estrogens promote cancer cell proliferation $(13,14)$.

The association between OATP4A1 expression and patient prognosis in a number of different types of tumor, especially that of CRC, and lung cancer, remains unclear (15). In a previous study, OATP4A1 abundance was reported to associate significantly with a decreased risk of tumor recurrence within 5 years. In the present study, patients with early stage CRC were investigated. In particular, high expression levels of OATP4A1 expression in tumor immune cells were revealed to associate with a decreased rate of early tumor recurrence (16). By contrast, another study by Ban et al (17) previously revealed that patients with more advanced CRC tumors exhibit increased levels of OATP4A1 in the tumor cells, have worse prognoses and decreased overall survival rates. These data suggest that OATP4A1 expression may serve different roles in the tumor cells and their microenvironment depending on the CRC stage.

Genetic variants in OATP4A1, which can alter the structure and function of the transporter, may also affect the susceptibility to CRC and/or alter the course of the disease. It is well known that $S L C O$ genes are characterized by a high genetic variability, with a minor allele frequency of $>5$ identified for OATP1B1, OATP1B3, and OATP1A2 (18). However, to the best of our knowledge, there is no information as yet on SLCO4A1 polymorphisms in patients with CRC compared with those in healthy controls. This is important as OATP variants have been documented to affect transporter properties, where single nucleotide polymorphisms (SNPs) identified in the transporter genes have been demonstrated to alter the expression and/or cellular localization of the protein (19). SNPs in the SLCO1B3 gene, which codes for OATP1B3, have been widely studied in CRC (20). It has been reported that the overexpression of OATP1B3 in CRC cells decreases the transcriptional activity of p53 and expression of its downstream transcription targets, cyclin-dependent kinase inhibitor 1 and p53-upregulated modulator of apoptosis (21). In particular, the OATP1B3 (SNP 583G $>$ E) variant, which lacks transport function, was not identified to exert any effect on the transcriptional activity of p53, suggesting that tumor inhibition is associated with transport activity (21). By contrast, 2 common SNPs in the SLCO1B1 (rs4149056 and rs2306283) and SLCO2B1 (rs2306168 and rs12422149) genes were not associated with CRC risk in patients (22). The role of the additional SNPs detected in 3 exons of the $S L C O 1 B 3$ gene in tumor samples from 30 patients treated for CRC in a Greek hospital remains unknown (23). In addition, even less is known about the role of the OATP4A1 variants in cancer physiology.

Although previous studies have reported the overexpression of OATP4A1 in CRC, to the best of our knowledge, the possibility that SNPs in the $S L C O 4 A 1$ gene can serve as a risk factor for CRC has not been investigated previously. Therefore, the present study aimed to determine whether the OATP4A1 variants were associated with an increased risk for CRC. Firstly, NCBI, dbSNP, and Ensembl databases were used to identify the most frequent non-synonymous mutations in the SLCO4AI gene. According to the Ensemble database, it was identified that 2 variants, G209>A (rs34419428, chr20:61288015) and G232>A (rs1047099, chr20:61288038), were the most frequent mutations present within the European population. In addition, the CRC variants and their association with the clinicopathological data, including sex, Union for International Cancer Control (UICC) stage 0/I vs. II and tumor recurrence following initial surgery within the follow-up period of $5 \pm 0.25$ years, were investigated in a sample of 178 patients with early stage $\mathrm{CRC}$ and 65 healthy controls. Paraffin-embedded CRC tissue samples were also used to determine if OATP4A1 abundance in the tumor samples is associated with these OATP4A1 genotypes. The functional activity of these OATP4A1 variants were studied in cells overexpressing them.

\section{Material and methods}

Patient samples. The present study was performed following the Declaration of Helsinki and the Ethical guidelines of the Institutions summarized in https://www.medunigraz.at/ethikkommission/index_dwnld.html.

Inclusion criteria for the present study were as follows: Patients with CRC stage 0/I and stage II primary tumors [classification according to the 7th edition of the TNM classification of the UICC (24)]; and aged 35-85 years. Patients were not excluded on sex. Follow-up for the patients was $5 \pm 0.5$ years following the primary surgical removal of the tumor.

Archived material from 178 patients of middle-European ethnicity with early stage CRC (77 females and 101 males; mean age $63.5 \pm 12.8$ years) were included into the present study. The guidelines of the Ethical Committee of the City of Vienna for retrospective studies (25) were applied. All patients 
had undergone surgery for primary CRC at the hospital Donauspital/Sozialmedizinisches Zentrum Ost between January 1992 and December 2009. In the cohort, stage 0/I tumors were present in 67 patients, whereas stage II tumors were present in 111 patients. Clinical data for a follow-up period of $5 \pm 0.25$ years were available for these patients. Relapse of the tumor either at the original site or at a distant site during follow-up was observed in 43 (25\%) of patients (mean time to relapse $809 \pm 164$ days). Patients with motality due to causes unrelated to CRC and patients with additional cancers were excluded from the present study.

From the surgically removed tumors, paraffin-embedded tissue blocks were routinely prepared at the Department of Pathology (Donauspital/Sozialmedizinisches Zentrum Ost) using neutral phosphate-buffered $10 \%$ formalin for fixation of the tissue for $16-24 \mathrm{~h}$ at room temperature. Serial sections $(4 \mu \mathrm{m})$ were cut from archived paraffin blocks, which were stored at $4^{\circ} \mathrm{C}$ at the Department of Pathology (Donauspital/Sozialmedizinisches Zentrum Ost). A section from each paraffin block was stained with Mayer's hematoxilin (Dako; Agilent Technologies GmbH) and eosin G-Lösung $0.5 \%$ (Sigma-Aldrich; Merck KGaA) for 3-5 min at room temperature following deparaffinization with xylene (three times), followed by treatment with ethanol gradients $(100 \%$ ethanol, twice for $3 \mathrm{~min}$; 95, 70 and 50\% ethanol for $3 \mathrm{~min}$, each) (26). Stained sections were then examined for tissue integrity and for the presence of areas enriched with cancer, immune, and stroma cells and normal mucosa adjacent to the tumor using a Zeiss AXIO Observer Light microscope (Zeiss, Jena, Deutschland) and the TissueFAXS Plus ${ }^{\circledR}$ cytometric system (TissueGnostics, Vienna, Austria) at a magnification, $\mathrm{x} 20$. Unstained sections were used for genotyping and immunohistochemistry.

DNA were obtained from 65 healthy volunteers of middle-European ethnicity (mean age, 33.5 \pm 8.2 years; 32: 33 females: males). DNA was extracted from blood cells provided by the Hungarian National Blood Transfusion Service and was used for genotype controls. Permission for using the samples for the study was obtained from the Medical Research Council of Hungary (https://ett.aeek.hu/en/secretariat/; 3027/2013). Informed consent was provided by the participants.

DNA isolation from formalin-fixed paraffin-embedded (FFPE) tissue sections. In the present retrospective study, DNA was isolated from the FFPE tissue sections, prepared from the archived tissue blocks, and then the frequency of the 2 OATP4A1 variants rs34419428 (G209>A) and rs1047099 (G232>A) was determined. Tissue scrapes were prepared using a razor blade from the sections, which were then collected into $1.5 \mathrm{ml}$ DNA LoBind tubes (Eppendorf). DNA was isolated using a NucleoSpin ${ }^{\circledR}$ FFPE XS DNA kit (Macherey-Nagel $\mathrm{GmbH} \& \mathrm{Co}$., $\mathrm{KG}$ ) according to the manufacturer's protocol. Briefly, a dissolver provided in the NucleoSpin ${ }^{\circledR}$ FFPE XS DNA kit was used to remove the paraffin from the FFPE tissue scrapes. Then, proteinase digestion was used to solubilize the fixed tissue and release DNA into solution. Heat incubation at $90^{\circ} \mathrm{C}$ for $30 \mathrm{~min}$ with the Decrosslink Buffer D-Link from the kit was then applied to eliminate crosslinks from the released DNA. Then, following the addition of ethanol, the lysate was applied to the NucleoSpin ${ }^{\circledR}$ DNA FFPE XS Column, where the DNA is bound to the silica membrane. Following 2 washing steps, the DNA was finally eluted under low ionic strength conditions in a small volume $(20 \mu \mathrm{l})$ of NucleoSpin ${ }^{\circledR}$ FFPE XS DNA kit Elution Buffer BE.

For each extraction, the DNA yields were quantified using a Qubit $^{\mathrm{TM}}$ 3.0 Fluorometer with the Quant-It kit (Thermo Fisher Scientific, Inc). The purity of the extracted nucleic acids was determined using the 260/280 $\mathrm{nm}$ absorbance ratios obtained using a NanoDrop ${ }^{\mathrm{TM}}$ spectrophotometer.

Restriction fragment length polymorphism (RFLP). To determine the presence of the G209>A (rs34419428, chr20:61288015; https://www.ncbi.nlm.nih.gov/snp/rs34419428) and G232>A (rs1047099, chr20:61288038; https://www.ncbi. nlm.nih.gov/snp/rs1047099) variants in CRC and control samples, the DNA fragments containing both SNPs were amplified by PCR using the following primers: Forward, 5'-GAC AAGCCGCTCACCTTC-3' and reverse 5'-CACAGGAAG AACAGGATGCC-3' (Invitrogen; Thermo Fisher Scientific, Inc.). PCR conditions ( 35 cycles) were as follows: Annealing of the primers at $55^{\circ} \mathrm{C}$ for $40 \mathrm{sec}$ and extension at $72^{\circ} \mathrm{C}$ for $1 \mathrm{~min}$. Prior to initiation of the 35 cycles, pre-incubation at $95^{\circ} \mathrm{C}$ was performed for $5 \mathrm{~min}$. The PCR fragment was digested with restriction enzymes Eco88I and/or Alw26I (Fermentas; Thermo Fisher Scientific, Inc.) to detect the presence of G209>A and G232>A, respectively, and subsequently analyzed on agarose gels after staining with SYBR Safe DNA Gel Stain (Invitrogen; Thermo Fisher Scientific, Inc.). Bands were documented and analyzed using the GelDoc Go Imaging System together with the Image Lab Touch 2.4 Software (Bio-Rad Laboratories, Inc.).

Haplotype frequencies and linkage-disequilibrium (LD) analysis. Haplotype frequencies were calculated by integrating the population-specific variant frequencies (Table SI) with the linkage information from the corresponding population (27). Data on the populations were provided by the 1000 Genomes Project using LDLink (28). To determine the LD, which gives the nonrandom association of alleles at different loci, for rs1047099 and rs34419428 in different ethnic groups, the LDpair analytical tool was used (https://analysistools.nci. nih.gov/LDlink/?var1=rs34419428\&var2=rs1047099\&pop=T SI\&tab=ldpair) (28).

Immunohistochemistry of FFPE sections. Immunohistochemical staining of tissue sections was performed using an affinity-purified anti-OATP4A1 polyclonal rabbit antibody (cat. no. HPA030669; Atlas Antibodies AB). If not stated otherwise, all steps were performed at room temperature $\left(22^{\circ} \mathrm{C}\right)$. In the preliminary experiments, serial dilutions of the antibody were examined to obtain optimal staining for OATP4A1 and minimize unspecific background staining of the tissue. Following de-paraffinization of the sections with xylene followed by treatment with an ethanol gradient (xylene, twice for $3 \mathrm{~min}$; xylene/ethanol mixture for $3 \mathrm{~min}$; $100 \%$ ethanol, twice for $3 \mathrm{~min} ; 95,70$ and 50\% ethanol for $3 \mathrm{~min}$, each), endogenous peroxidase activity was blocked using $0.3 \% \mathrm{H}_{2} \mathrm{O}_{2}$ in PBS for $10 \mathrm{~min}$. Repeated washing (three times) with PBS was performed, followed by a blocking step for unspecific binding sites on the antigen using the horseradish 
peroxidase polymer blocking solution provided in the Thermo Scientific Lab Vision ${ }^{\mathrm{TM}}$ UltraVision $^{\mathrm{TM}}$ LP Detection System (Thermo Fisher Scientific, Inc.) for $1 \mathrm{~h}$ at room temperature. The UltraVision ${ }^{\mathrm{TM}}$ LP detection system contains a universal secondary antibody formulation conjugated to an HRP-labeled polymer that recognizes mouse and rabbit IgG. To detect OATP4A1, the HPA030669 antibody (Atlas Antibodies AB; 1:50 dilution in PBS containing $0.5 \%$ bovine serum albumin) was applied at $4^{\circ} \mathrm{C}$ overnight. Following repeated washing, the UltraVision ${ }^{\mathrm{TM}}$ LP antibody enhancer solution containing goat anti-rabbit IgG was added for $1 \mathrm{~h}$. The UltraVision ${ }^{\mathrm{TM}} \mathrm{LP}$ Detection System HRP polymer and DAB Plus Chromogen were then used for the detection of OATP4A1 staining. Finally, cell nuclei were stained using water-based Mayer's hematoxylin solution (Dako; Agilent Technologies $\mathrm{GmbH}$ ) for $1 \mathrm{~min}$ and sections were mounted using dibutylphthalate polystyrene xylene mounting medium (Sigma-Aldrich; Merck KGaA).

Quantitative image cytometry of the FFPE sections. The TissueFAXS Plus ${ }^{\circledR}$ cytometric system (TissueGnostics GmbH) equipped with an AXIO Imager Zeiss 1 automated light microscope (Carl Zeiss AG) and the HistoQuest ${ }^{\circledR}$ software (version 4.0.4.150; TissueGnostics $\mathrm{GmbH}$ ) was used for image acquisition at magnification, x20, and quantitative evaluation of OATP4A1-positive cells as described previously (29). To determine the proportion of OATP4A1-positive cells, the gray values of the DAB signal intensity were assessed after correcting for the background values from the negative control samples, where the primary antibody was replaced with non-immunogenic IgG. The proportions of OATP4A1-positive tumor, immune, stroma, and adjacent mucosa cells were calculated from the total number of OATP4A1-positive tumor, immune, stroma, and adjacent mucosa cells per area, respectively (set to $100 \%$ ).

The blue color of hematoxylin-stained nuclei allows for an estimation of tissue quality and staining efficacy of the procedure in the individual samples. From the original cohort, samples from 6 patients were excluded due to the poor morphological preservation of the tissue, which did not result in a staining pattern that was acceptable for the automated analysis by the FAXS system. Analysis was performed on sections from 172 patients on defined regions of interest covering the following areas in the tumor sample: Cancer cell-enriched areas and the surrounding stroma; infiltrating immune cells; and morphological normal mucosa cells as described previously $(29,30)$.

Studies on the G209>A (rs34419428) and G232>A (rs1047099) SLCO4A1 variants in Sf9 insect cells and A431 cancer cell lines

Generation of the Sf9 and A431 cells overexpressing the OATP4Al variants. Generation of the plasmid construct for expressing OATPs in Sf9 cells was performed as described previously (9). The baculovirus transfer vector pAcUW21 (BD Biosciences) was engineered to give the modified plasmid pAcUW-L2, which is suitable for cloning of all OATPs (9). Full-length cDNA sequences encoding human OATP4A1 variants were introduced into the pAcUW-L2 vector. Sequencing of the OATP4A1 construct from the Harvard Plasmid ID Repository (Harvard Medical School) revealed that the cDNA contained the 232G $>$ A SNP (V78I, rs1047099). Therefore, the canonical sequence for wild-type (wt) OATP4A1 (HGNC: 10953; Entrez Gene: 28231; Ensembl: ENSG00000101187; OMIM: 612436; UniProtKB: Q96BD0-1) was generated using the QuikChange Mutagenesis kit (Thermo Fisher Scientific, Inc.). Also, other probes for the variants R70Q and R70Q/V78I (GenBank accession numbers: variant rs34419428, NM_016354.4: c.209G>A; NP_057438.4: p.R70Q and variant rs1047099, NM_016354.4: c.232G>A; NP_057438.4: p.V78I; Table SI) were generated by PCR site-directed mutagenesis. The following primers were used: Wt forward, 5'-AGGTGC GGTACGTCTCGG-3'; Wt reverse, 5'-CCGAGACGTACC GCACCT-3'. R70Q forward, 5'-GCCCAGGGGACCCAT GA-3'; R70Q reverse, 5'-TCATGGGTCCCCTGGGC-3'; R70Q/V78I forward, 5'-GCCCAGGGGACCCATGA-3'; and R70Q/V78I reverse, 5'-TCATGGGTCCCCTGGGC-3'. The PCR reaction was performed using $2.5 \mathrm{U} / \mu 1$ Phusion High-Fidelity DNA Polymerase (Thermo Fisher Scientific, Inc.). DNA sequences of all the constructs were verified using the DNA Sanger sequencing method. At least 2 clones were sequenced from each plasmid.

The human epidermoid carcinoma A431 cell line was obtained from ATCC and cultured in DMEM supplemented with $10 \%$ FBS (Gibco; Thermo Fisher Scientific, Inc.). Cells were maintained at $37^{\circ} \mathrm{C}$ in a humidified atmosphere under $5 \%$ $\mathrm{CO}_{2}$. Wt and variant OATP4A1-overexpressing A431 cells were generated by the transposase (Thermo Fisher Scientific, Inc.) mediated genomic insertion method (31) using a pSB-CMV vector (Promega Corporation). For transfection, the PCR fragments were cloned between the NotI-HindIII (New England Biolabs Inc.) sites of the pSB-CMV vector $(29,30)$. Cell selection was performed with $1 \mu \mathrm{g} / \mathrm{ml}$ puromycin (Sigma-Aldrich; Merck KGaA). Thereafter, the cells were maintained in DMEM (Gibco, Thermo Fischer) supplemented with 10\% FBS, 2 mM L-glutamine, $100 \mathrm{U} / \mathrm{ml}$ penicillin, and $100 \mu \mathrm{g} / \mathrm{ml}$ streptomycin (Sigma-Aldrich; Merck $\mathrm{KGaA}$ ) at $37^{\circ} \mathrm{C}$ with $5 \% \mathrm{CO}_{2}$ and $95 \%$ humidity $(32,33)$.

Sf9 cells from Spodoptera frugiperda were obtained from ATCC (Sf9 ATCC ${ }^{\circledR}$ CRL-1711 ${ }^{\mathrm{TM}}$ ) and were grown in a 0.5 liter round-bottom spinner flask in TNM-FH insect medium (Sigma-Aldrich; Merck KGaA), supplemented with 10\% FBS, $100 \mathrm{U} / \mathrm{ml}$ penicillin and $100 \mu \mathrm{g} / \mathrm{ml}$ streptomycin (Sigma-Aldrich; Merck KGaA) at $27^{\circ} \mathrm{C}$. These cells were previously used to study OATP-mediated transport (34). Recombinant baculoviruses, carrying the different human $S L C O 4 A 1$ gene sequences, were generated using the BD BaculoGold ${ }^{\mathrm{TM}}$ Transfection kit (BD Biosciences), according to the manufacturer's protocols, and were stored at $4^{\circ} \mathrm{C}$.

Western blot analysis. Western blot analysis was used to detect the expression of the wtOATP4A1, and the polymorphic variants expressed in Sf9 and A431 cells. All the following isolation steps were done at $4^{\circ} \mathrm{C}$ to obtain the proteins. Cells were suspended in $50 \mathrm{mM}$ Tris/HCL buffer, $\mathrm{pH} 7.0$, containing $50 \mathrm{mM}$ mannitol, $2 \mathrm{mM}$ EGTA, $10 \mathrm{pg} / \mathrm{ml}$ leupeptin, $8 \mathrm{pg} / \mathrm{ml}$ aprotinin, $0.5 \mathrm{mM}$ phenylmethylsulfonyl fluoride and $2 \mathrm{mM}$ beta-mercaptoethanol (all from Sigma-Aldrich; Merck KGaA). Thereafter, cells in this suspension buffer were homogenized to obtain a homogenate containing cells, organelles and membranes. However, it contained also some undisrupted 
cells and larger cell debris. To remove undisrupted cells and larger debris from the homogenate, the sample was centrifuged at $500 \mathrm{x} \mathrm{g}$ for $10 \mathrm{~min}$, followed by a centrifugation step at 2,000 $\mathrm{x}$ g for $10 \mathrm{~min}$ to remove mitochondria and other cell organelles, such as lysosomes. Finally, a crude membrane fraction was recovered by centrifugation $(60 \mathrm{~min}$ at $100,000 \mathrm{x} \mathrm{g})$. This pellet containing the proteins was resuspended in the homogenization buffer to obtain a protein concentration of $2 \mathrm{mg} / \mathrm{ml}$. All procedures were carried out at $4^{\circ} \mathrm{C}$ and samples were stored at $-70^{\circ} \mathrm{C}$ for further applications. Protein concentration was determined using modified Lowry method (Sigma-Aldrich; Merck KGaA).

Proteins $(25 \mu \mathrm{g})$ were separated by $4-15 \%$ SDS-PAGE and transferred onto PVDF membranes (Bio-Rad Laboratories, Inc.). After blocking unspecific binding sites on the protein with the Invitrogen ${ }^{\mathrm{TM}}$ membrane blocking solution (Thermo Fisher Scientific, Inc.) for $1 \mathrm{~h}$ at room temperature, membranes were incubated with the polyclonal anti-OATP4A1 antibody overnight at $4^{\circ} \mathrm{C}(1: 1,000)$. The antibody was provided by Dr. Bruno Stieger, (Division of Clinical Pharmacology and Toxicology, Universitätsspital Zürich, Switzerland) or non-immunogenic IgG (Thermo Scientific ${ }^{\mathrm{TM}}$ Lab Vision $^{\mathrm{TM}}$ ) for the negative control. Following primary antibody incubation, the membranes were incubated with a horseradish (HRP)-conjugated anti-rabbit secondary antibody at a dilution of 1:10,000 for $1 \mathrm{~h}$ at room temperature (cat. no. AB_2307391; Jackson ImmunoResearch Europe, Ltd.). Immunoreactive protein bands were detected using the Pierce ${ }^{\mathrm{TM}}$ ECL Western Blotting Substrate (Thermo Fisher Scientific, Inc.).

Immunofluorescence staining. A431 cells overexpressing mutant or wtOATP4A1 cultured on cover slides were fixed using methanol (stored at $-20^{\circ} \mathrm{C}$ ) for $5 \mathrm{~min}$. Following repeated washing and blocking of unspecific binding sites with $10 \%$ bovine serum albumin (Sigma-Aldrich; Merck KGaA) for $1 \mathrm{~h}$, the antibody against OATP4A1 (cat. no. HPA030669, Atlas Antibodies AB) was diluted 1:100 in PBS containing 2\% rabbit serum (Vector Laboratories; Maravai LifeSciences) and applied overnight. Prior to the staining procedure, optimal antibody concentrations were determined by titrating serial antibody dilutions. The applied dilution corresponds to the minimum concentration necessary to produce a positive signal. Following primary antibody incubation, a secondary antibody (Alexa Fluor 488-anti-rabbit IgG; cat. no. AB_2338052; Jackson ImmunoResearch Europe Ltd.), diluted 1:500 in PBS was applied for $1 \mathrm{~h}$ at room temperature. For staining of the nuclei, DAPI (Roche Diagnostics) was used at a concentration of $0.2 \mu \mathrm{g} / \mathrm{ml}$ for $10 \mathrm{~min}$ at room temperature. In the control experiments, the primary antibody was replaced by non-immunogenic IgG (Thermo Fisher Scientific Lab Vision ${ }^{\mathrm{TM}}$; Thermo Fisher Scientific, Inc.). No staining for OATP4A1 was observed. Finally, following repeated washing, the slides were mounted in Fluoromount ${ }^{\mathrm{TM}}$ mounting medium (Sigma-Aldrich; Merck KGaA) prior to visualization using the LSM 900 Confocal Laser Scanning Microscope (Carl Zeiss AG; magnification, $\mathrm{x} 20$ ).

Uptake of sodium-fluorescein in Sf9 and A431 cells overexpressing OATP4A1 and its polymorphic variants. Transport experiments in $\mathrm{Sf} 9$ and $\mathrm{A} 431$ cells were performed using the fluorescent OATP substrate, sodium fluorescein, as previously described (35). Briefly, $5 \times 10^{5}$ cells were incubated with $1 \mu \mathrm{M}$ sodium fluorescein (Sigma-Aldrich, Merck KG) at pH 5.5 for $10 \mathrm{~min}$. The reaction was stopped using $1 \mathrm{ml}$ ice-cold PBS. The cellular fluorescence was measured at the emission wavelength of $530 \mathrm{~nm}$ following the excitation wavelength of $488 \mathrm{~nm}$ using an Attune ${ }^{\mathrm{TM}}$ acoustic focusing cytometer (Thermo Fisher Scientific, Inc.).

Position of the two SNPs in a two-dimensional (2D) structure of OATP4A1. The 2D structure of human OATP4A1 has been determined using the HMMTOP transmembrane topology prediction server; 2.0 version (36). The diagram was drawn according to the information available at: http://emboss.bioinformatics.nl/cgi-bin/emboss (version: EMBOSS:6.6.0.0).

Statistical analysis. Statistical analysis was performed using SAS software (version 9.4; SAS Institute, Inc.), and categorical variables are presented as counts and percentages.

Clinical data from the 172 patients including sex, UICC stage $0 / I$ and II, relapse within $5 \pm 0.25$ years, were used for the statistical calculations. Statistical comparisons between groups were performed using $\chi^{2}$ tests, whilst Cramer's V was used to quantify the associations between categorical variables. For Cramer's V, values range between +1 for perfect agreement and -1 for perfect disagreement. A Kruskal-Wallis test was used to compare the percentages of OATP4A1-positive cells between tumor (tumor, immune, and stroma cells) and the adjacent mucosa cells. Within each OATP4A1 variant, multiple comparisons among the three cell types in the tumor and the tumor-adjacent mucosa cells were corrected by the Bonferroni-Holm adjustment. $\mathrm{P} \leq 0.05$ was considered to indicate a statistically significant difference.

\section{Results}

OATP4A1 allele frequency in patients with CRC and controls. The frequency of the two most common missense mutations in the SLCO4Al gene in the European population, rs34419428; c. $209 \mathrm{G}>\mathrm{A}$, which encodes the polymorphic variant R70Q, and rs1047099; c. 232G>A, which encodes the R78I variant, were assessed (Table SI). The structure of rodent Oatp1 was used as a template to illustrate the position of the mutant amino acids in the two OATP4A1 variants (37). According to computer-based hydropathy analysis, all of the OATP4A1 variants were identified to share a similar transmembrane domain organization, with 12 predicted transmembrane domains, where the $\mathrm{N}$ - and C-terminal ends are located on the cytoplasmic side of the membrane. The sequence defining the OATP superfamily is located on the boundary of the extracellular loop 3 and transmembrane domain 6. The two SNPs investigated in the present study were identified to be in the first intracellular loop of the cytoplasmic domain (Fig. 1).

Genotyping of the 2 variants was conducted by RFLP using DNA from 178 patients with CRC and 65 healthy controls. A frequency of 0.038 was observed for the R90Q allele and 0.292 for the V78I allele in patients with CRC, whilst similar frequencies of 0.051 and 0.286 , were observed for the R90Q and the V78I alleles, respectively, in the control group (Table I). Statistical analysis revealed that there was no significant difference between the genotype frequency in the 


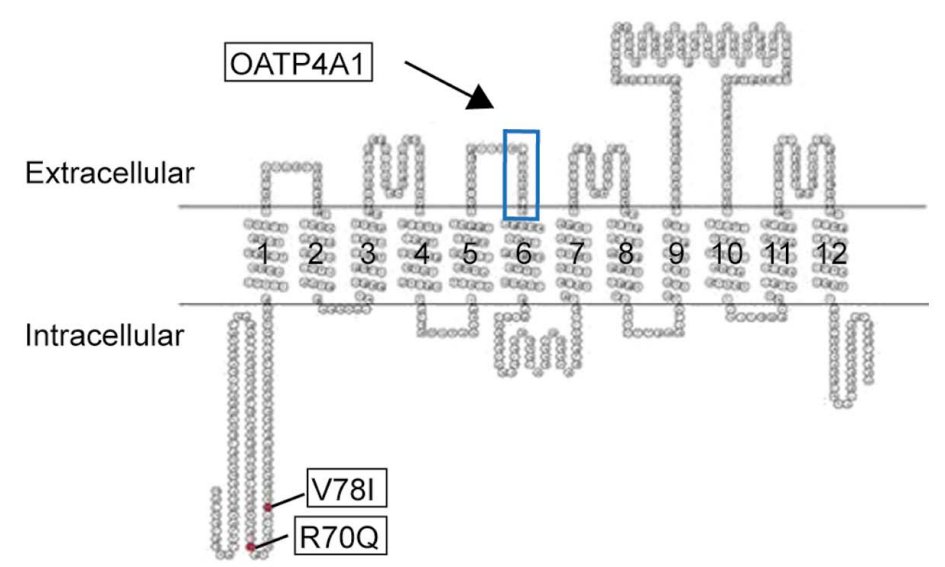

Figure 1. Predicted membrane topology of OATP4A1 and the position of the 2 non-synonymous rs34419428 and rs1047099 polymorphisms in the protein structure. Structural mapping of $S L C O 4 A 1$ variants was performed using the program on EMBOSS. Domains are orientated from the $\mathrm{N}$ - to the C-terminus of the polypeptide. The 2 SNPs are separated by 8 amino acids and are located in the first intracellular loop. The OATP signature [D-X-RW-(I/V)-GAWW-X-G$(\mathrm{F} / \mathrm{L})-\mathrm{L}]$ is located on the 3rd extracellular loop next to transmembrane region 6, which was indicated by the arrow. Transmembrane regions 1-10 are important for substrate recognition. OATP4A1, solute carrier organic anion transporter family member 4A1; SLCO, solute carrier for organic anion; SNP, singe nucleotide polymorphism.

control and $\mathrm{CRC}$ groups $(\mathrm{P}=0.56$ and $\mathrm{P}=0.52$ for the $\mathrm{R} 90 \mathrm{Q}$ and the V78I alleles, respectively).

The linkage equilibrium between the two alleles in the mixed European population was next determined by the LDpair analytical tool using data for the R90Q and the V78I allele frequency from the 1000 genome project (Table SI). The statistical analysis for the random association of the two alleles in the different populations revealed a value of $\mathrm{D}^{\prime}=1$, suggesting a high level of allelic association. The $\mathrm{r}^{2}$ value of 0.12 and the high $\chi^{2}$ value of $120(\mathrm{P}<0.0001)$ further suggested that these two alleles are associated with each other (Table SII).

All individuals in the CRC and control groups were identified to carry either hetero- or homozygous mutations for the V78I allele, but were always heterozygous for the R70Q allele (Table I). No significant difference was observed between the patients with CRC and controls in the distribution of these two minor alleles. In the CRC group, $7.7 \%$ of patients were heterozygous for the R90Q allele and $46.2 \%$ for the V78I allele. Similarly, in the control group, $10.2 \%$ of individuals were heterozygous for the R70Q allele and 39.0\% for the V78I allele. There was also no difference between the CRC and the control group regarding the proportion of V78I homozygous individuals. $9.1 \%$ individuals were homozygous in the CRC and $6.2 \%$ in control groups, respectively (Table I).

OATP4A1 variants and patient characteristics. The association between the 2 polymorphic variants of OATP4A1 in patients with CRC and their respective clinicopathological characteristics [female $(n=74)$ and male $(n=98)$; UICC stage 0/I $(\mathrm{n}=61)$ and UICC stage II $(\mathrm{n}=111)$; relapse within the follow-up period of $5 \pm 0.25$ years $(n=43)$ and no relapse $(n=129)$ ] was analyzed using the Cramer's V and $\chi^{2}$ test. From these 172 patients, FFPE sections were available for the quantitative analysis of OATP4A1 expression. No association between the parameter was observed. Data are summarized in Table II.

In addition, this analysis was performed to study whether haplotypes are associated with these parameters. The 3 haplotypes were examined as a collective (Tables SI and SII). Type 1 consists of wtR70 and wtV78 with nucleotides $\mathrm{G}$ and $\mathrm{G}$ in the two alleles at amino acid position 70 and 78 , respectively; type 2 consists of wtR70 and mutant V78I resulting from the nucleotides being $\mathrm{G}$ and $\mathrm{A}$, respectively; and type 3 has the R70Q and V78I alleles encoded by the $\mathrm{A}$ and $\mathrm{A}$ nucleotide variations, respectively.

The type 4 haplotype with R70Q and wtV78 (nucleotides A and $\mathrm{G}$ in the respective allele) was not present in the samples examined in the present study, consistent with data from the 1000 Genomes project on the haplotype distribution in the Middle European population. Further data on the distribution of the haplotypes among different ethnic groups are provided in detail in the Tables SI and SII. It was observed that Type 4 is present only in ethnic groups from Africa.

The association between haplotypes and clinicopathological data is summarized in Table II. Values obtained for Cramer's V ranged between-0.09 and 0.09, which indicated that there was no significant correlation between the clinicopathological parameters and the haplotypes. However, independence could not be rejected according to the results from the $\chi^{2}$ test $(\mathrm{P}=0.08-0.87)$.

OATP4A1 variants in CRC tumor sections. To determine if the percentage of OATP4A1-positive cells in the CRC tumors is associated with the polymorphic variants in the CRC patients, the FFPE tumor sections were stained for OATP4A1. Controls were stained with non-immunogenic IgG. On the tissue sections, positive staining for OATP4A1 was observed in the cytosol and on the membrane of tumor cells, while the membrane vs. cytosol localization was not identified to be significantly different among patients carrying the wtOATP4A1 or those harboring variants (Fig. 2). In adjacent mucosa cells, positive staining for OATP4A1 was almost always (>95\%) observed to be located on the membrane.

An automated quantitative microscopic image analysis system was used to determine the number of OATP4A1-positive cells in areas enriched in cancer, immune, and stroma cell and the mucosa adjacent to the tumor (Fig. 2A and B). Following correction for the unspecific background staining of the samples, the percentage of positive cells per area was then calculated. The portion of OATP4A1-positive cells in all areas 
Table I. Wild-type OATP4A1 and the V78I and R90Q minor alleles in patients with colorectal cancer and in healthy individuals as controls.

\begin{tabular}{|c|c|c|c|c|}
\hline \multirow[b]{2}{*}{ Individuals } & \multicolumn{2}{|c|}{ Controls $(n=65)$} & \multicolumn{2}{|c|}{ Patients with CRC $(n=187)$} \\
\hline & R70Q & V78I & R70Q & V78I \\
\hline Minor alleles, $\mathrm{n}(\%)$ & $5(3.8)$ & $38(29.2)$ & $19(5.1)$ & $107(28.6)$ \\
\hline Minor allele, frequency & 0.038 & 0.292 & 0.051 & 0.286 \\
\hline Wt individuals, $\mathrm{n}(\%)$ & $60(92.3)$ & $31(47.7)$ & $168(89.8)$ & $97(51.9)$ \\
\hline Heterozygous individuals, n (\%) & $5(7.7)$ & $30(46.1)$ & $19(10.2)$ & $73(39.0)$ \\
\hline Homozygous individuals, n (\%) & $0(0.0)$ & $4(6.2)$ & $0(0.0)$ & $17(9.1)$ \\
\hline Non-wt individuals, $\mathrm{n}(\%)$ & $5(7.7)$ & $34(52.3)$ & $19(10.2)$ & $90(48.1)$ \\
\hline Chromosomes, $\mathrm{n}$ & 130 & 130 & 374 & 374 \\
\hline
\end{tabular}

Genotyping was performed in the patients with CRC and in healthy individuals (controls). The total number (n) of individuals in each group was taken as $100 \%$. No significant statistical difference was observed between the allele distribution in patients with CRC and controls $\left(\chi^{2}\right.$-test; $\mathrm{P}=0.34-0.99)$. CRC, colorectal cancer.

Table II. Association between variant OATP4A1 and clinicopathological data from patients with colorectal carcinoma.

Clinicopathological data

\begin{tabular}{|c|c|c|c|c|c|c|}
\hline \multirow[b]{2}{*}{ OATP4A1 } & \multicolumn{2}{|c|}{$\begin{array}{c}\text { Sex, } \mathrm{n} \\
\text { (female:male, } 74: 98 \text { ) }\end{array}$} & \multicolumn{2}{|c|}{ UICC Stage, n 0/I:61/II:111 } & \multicolumn{2}{|c|}{ Relapse, n yes:43/no:129 } \\
\hline & Cramer's V & $\mathrm{P}$-value & Cramer's V & P-value & Cramer's V & P-value \\
\hline R70Q & -0.09 & 0.26 & -0.02 & 0.75 & -0.11 & 0.15 \\
\hline V78I & 0.07 & 0.63 & 0.13 & 0.21 & 0.17 & 0.08 \\
\hline Haplotype & 0.09 & 0.47 & 0.07 & 0.65 & 0.11 & 0.33 \\
\hline V78I_dom & -0.06 & 0.40 & -0.07 & 0.35 & -0.07 & 0.38 \\
\hline V78I_rec & 0.01 & 0.87 & 0.08 & 0.28 & 0.12 & 0.10 \\
\hline
\end{tabular}

Associations between the OATP4A1 variants and the clinicopathological parameter of the patients were calculated using Cramer's V. Data were also analyzed assuming a dom and a rec V78I allele. The $\chi^{2}$ test was used to determine P-values. OATP4A1, solute carrier organic anion transporter family member 4A1; dom, dominant; rec, recessive.

was identified to be independent of whether the patients carried the wtOATP4A1 or the polymorphic variants (Table III).

Studies in Sf9 and A431 cells overexpressing OATP4A1 variants. To determine whether the SNPs resulted in changes in the expression levels, localization, or function of OATP4A1, Sf9 (S. frugiperda) cells and A431 cell lines overexpressing either wtOATP4A1 or its R70Q, V78I or R70Q/V78I variants were established. Sf9 cells provide a well-known eukaryotic expression system that is able to produce large amounts of active recombinant proteins such as transporters, and is useful for investigating the biochemical and pharmacological properties of the proteins expressed (34). These cells have also been successfully used in the pharmaceutical industry for studying drug uptake and excretion pharmacologically. However, unlike transfected human cell lines, Sf9 cells cannot glycosylate proteins. Therefore, the human skin tumor A430 cell line was also transfected and expresses sufficient wt and variant OATP4A1 required for transportation studies $(9,35)$.
The expression of wtOATP4A1 or their variants were first analyzed using western blot analysis and immunofluorescence staining (Fig. 3). According to the western blot analysis results, the anti-OATP4A1 antibody produced a single immunoreactive band at $\sim 70 \mathrm{kDa}$ for both wt and the variant OATP4A1. The subcellular localization of the transporter was next studied in A431 cells transfected with wtOATP4A1 or the variants. Following immunofluorescence staining using Alexa Fluor ${ }^{\circledR}$ 488-labeled antibodies, green fluorescence, representing labelled OATP4A1 proteins, was visible on the cell membranes of the cells transfected with wt or OATP4A1 variants (Fig. 3).

Following the validation of the expression of the variants, their capability of transporting the sodium fluorescein, a model substrate used for studying OATP-mediated transport, was determined (32). An increased accumulation (2-3-fold) of the fluorescent dye was observed in Sf9 and A431 cells transfected with the wt or OATP4A1 variants compared with that in cells transfected with the empty plasmid. Importantly, 
Table III. Statistical analysis of differences in the proportion of OATP4A1-positive cells in tumor sections derived from patients with CRC with variant OATP4A1.

\begin{tabular}{|c|c|c|c|}
\hline OATP4A1 variant & OATP4A1-positive cells ${ }^{\mathrm{a}}$ & P-value & P-value (corr $\left.{ }^{b}\right)$ \\
\hline \multirow[t]{4}{*}{ R70Q } & Immune cells & 0.628 & 1.000 \\
\hline & Tumor cells & 0.046 & 0.185 \\
\hline & Adjacent mucosa & 0.344 & 1.000 \\
\hline & Stroma cells & 0.548 & 1.000 \\
\hline \multirow[t]{4}{*}{ V78I } & Immune cells & 0.619 & 1.000 \\
\hline & Tumor cells & 0.161 & 0,644 \\
\hline & Adjacent mucosa & 0.759 & 1.000 \\
\hline & Stroma cells & 0.256 & 0.767 \\
\hline \multirow[t]{4}{*}{ Haplotype } & Immune cells & 0.402 & 0.804 \\
\hline & Tumor cells & 0.096 & 0.385 \\
\hline & Adjacent mucosa & 0.440 & 0.804 \\
\hline & Stroma cells & 0.267 & 0.802 \\
\hline \multirow[t]{4}{*}{ V78I_dominant } & Immune cells & 0.329 & 0.659 \\
\hline & Tumor cells & 0.129 & 0.417 \\
\hline & Adjacent mucosa & 0.624 & 0.659 \\
\hline & Stroma cells & 0.104 & 0.417 \\
\hline \multirow[t]{4}{*}{ V78I_recessive } & Immune cells & 0.786 & 1.000 \\
\hline & Tumor cells & 0.586 & 1.000 \\
\hline & Adjacent mucosa & 0.687 & 1.000 \\
\hline & Stroma cells & 0.395 & 1.000 \\
\hline
\end{tabular}

OATP4A1 abundance was analyzed in immunohistochemically stained tumor sections from patients with wt and variant OATP4A1. ${ }^{\mathrm{a} T h e}$ proportion of OATP4A1-positive tumor, immune, stroma, and adjacent mucosa cells, was calculated from the total number of OATP4A1-positive tumor, immune, stroma, and adjacent mucosa cells, respectively, per area (100\%). Kruskal-Wallis tests were used to compare the proportion of OATP4A1-positive cells between groups. Corr ${ }^{\mathrm{b}}$, values were corrected for multiple testing. CRC, colorectal cancer; OATP4A1, solute carrier organic anion transporter family member $4 \mathrm{~A} 1$.
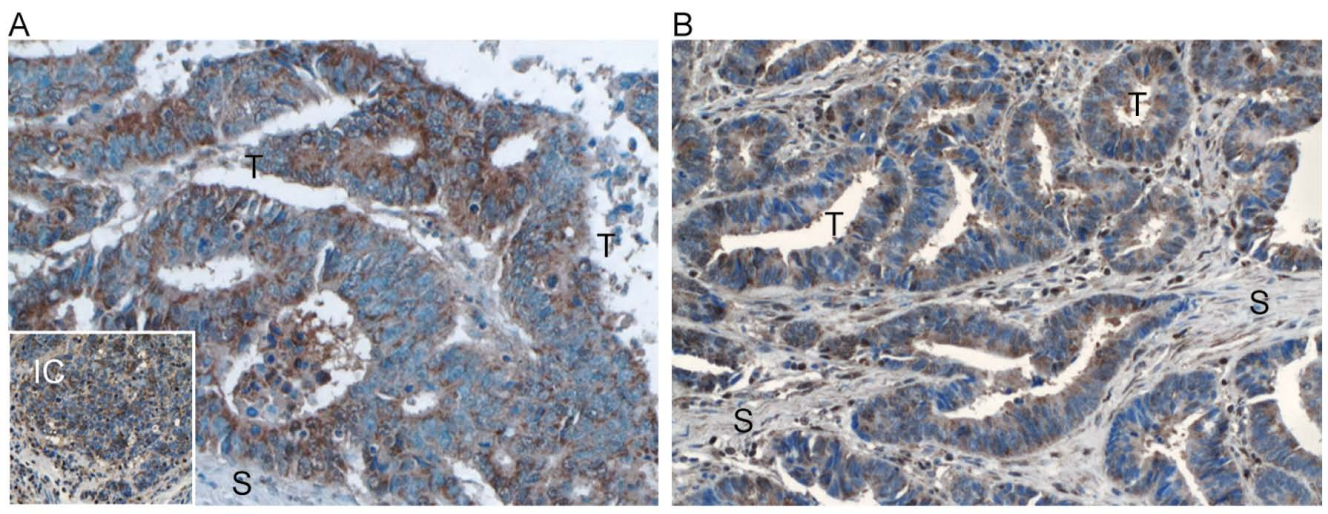

Figure 2. Immunohistochemical staining of CRC tissue sections from patients with Wt and mutant OATP4A1. (A) Representative image of a tumor section from a patient with wtOATP4A1 using a rabbit polyclonal antibody against OATP4A1 for staining. Insert represents IC in the tumor. Original magnification, x20. (B) Representative image of a tumor sample from a patient expressing the OATP4A1 variant V78I. Magnification, x20. wt, wild-type; OATP41, solute carrier organic anion transporter family member 4A1; CRC, colorectal cancer; T, tumor cells; S, stroma; IC, immune cells.

no significant difference was identified between the transport function of wtOATP4A1 and that of the variants (Fig. 4).

\section{Discussion}

OATPs have been previously identified to exhibit significant genetic variability (18), and polymorphisms in these transporters may affect cancer risk and progression, drug responses and the outcome of subsequent therapeutic efficacy $(38,39)$. To date, the most commonly studied polymorphisms in the $S L C O$ genes are those encoding for the OATP1 family $(19,40)$. SNPs present in OATP1B3 and OATP1B1 have been identified to exert severe side effects due to the altered uptake kinetics of anticancer drugs such 
A

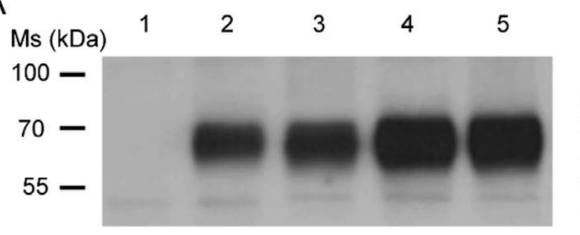

Lanes:

1: Sf9 control

2-5: Sf9 with

2: wt OATP4A1

3: R70Q

4: $V 781$

5: V78I/R70Q

B

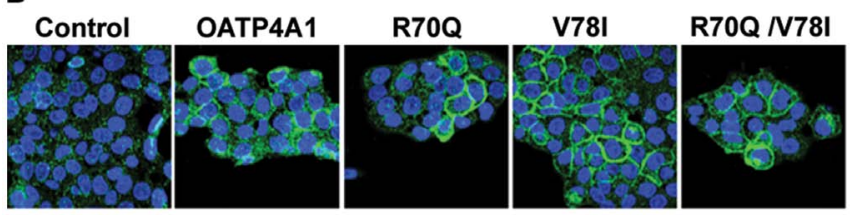

Figure 3. OATP4A1 (wt, R70Q, V78I, and R70Q/V78I variant) overexpression in Sf9 insect and A431 cancer cells. (A) Western blot of Wt and variant OATP4A1 in Sf9 insect cells probed with a polyclonal anti-OATP4A1 antibody. There were $10 \mu \mathrm{g}$ whole Sf9 cell lysates were analyzed. Lane $1, \mathrm{sF} 9$ cells with the empty plasmid (control); lanes 2-5, sF9 cells overexpressing either wt OATP4A1, (shown in lane 2); or variant OATP4A1 (lanes 3-5). Variant R70Q is shown in lane 3, V78I in lane 4 and V78I/R70Q in lane 5. (B) Confocal images of A431 cells transfected with the empty vector (control) and cells overexpressing wt OATP4A1 and the R70Q, V78I or R70Q/V78I variants. Magnification, x20. Nuclei were stained blue using DAPI. wt, wild-type; OATP4A1, solute carrier organic anion transporter family member 4A1; CRC, colorectal cancer; Ms, molecular mass.

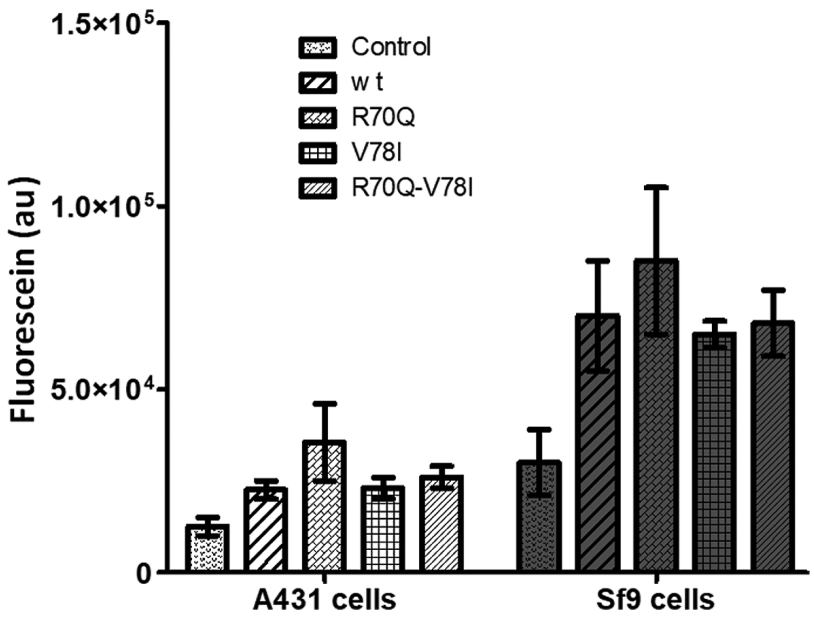

Figure 4. Accumulation of sodium-fluorescein in Sf9 and A431 cells overexpressing the wt OATP4A1 or the SNP variants. Transport experiments were performed using $1 \mu \mathrm{M}$ OATP4A1 surrogate substrate, sodium-fluorescein. Sf9 and A431 cells transfected with the empty plasmid were used as negative controls. Data are presented as the mean \pm SD $n=3$. OATP4A1, solute carrier organic anion transporter family member 4A1; SNP, single nucleotide polymorphism; au, arbitrary units; wt, wild type.

as that of irinotecan, which is frequently used for treating CRC $(41,42)$.

Although a previous study observed that OATP4A1 was overexpressed in CRC, to the best of our knowledge, no data are available regarding the effect of SNPs in genes encoding for OATP4A1 on the outcome or progression of CRC. Studies on OATP4A1 expression levels and its role in the altered pharmacokinetic activity of drugs are limited. Benzylpenicillin and an isoprostane metabolite have been previously identified as OATP4A1 substrates in vitro (43). Notably, methotrexate (MTX), which exerts antiproliferative and cytotoxic effects in a number of cell types such as colon cancer cells, was proposed to be OATP4A1 substrate. It was investigated whether the presence of the polymorphic OATP4A1 variants would alter the outcome of a specific therapy against rheumatoid arthritis (44). MTX is an agent that is frequently used in cancer chemotherapy and an established treatment strategy for autoimmune and inflammatory diseases such as rheumatoid arthritis. In a study in patients with arthritis, a $\mathrm{C}>\mathrm{T}$ intron variant (rs2236553) with a frequency of 0.34 in the European population was identified to affect the efficacy of MTX treatment and promote drug toxicity (44). By contrast, a second study did not confirm these results (45). In fact, it suggested that MTX was unlikely to be a substrate for OATP4A1. The cellular accumulation of MTX may be caused by the action of other OATPs, including OATP1B1 and OATP1B3 (45).

The expression levels of OATP4A1 have been reported to be important for CRC predisposition and tumor progression $(16,17)$. Therefore, the present study aimed to elucidate whether variants in OATP4A1 may predispose individuals to CRC. A total of 2 frequent nonsynonymous polymorphisms in OATP4A1 (rs34419428 and rs1047099) were investigated in 178 patients with CRC and 65 healthy controls of Austrian and Hungarian origin. It was identified that $<50 \%$ of all individuals were heterozygous for the c.232G $>$ A allele (p.V78I) and 7-10\% for the c.209G $>$ A (p.R70Q) allele. These results on the allelic frequency in the $\mathrm{CRC}$ patients and controls were consistent with data involving a mixed European population, which were previously published in the 1000 Genomes Project (46). Notably, all individuals harboring the 209A (R70Q) allele also had the minor allele c. $232 \mathrm{G}>\mathrm{A}$. As both SNPs are located in the same exon, they can be co-amplified by one PCR reaction. Using RFLP-PCR, it was shown that the R70Q minor allele (rs34419428) is associated with the V78I minor allele (rs1047099) in the genome of patients with CRC and control individuals in the present cohort.

In the present study, no association was identified between the sex, UICC stage and tumor recurrence of patients with early stage CRC and the 2 OATP4A1 polymorphic variants. The distribution of the 2 alleles differs among different ethnic groups, but none of the polymorphic genotypes in the present cohort were associated with the CRC risk and the risk a tumor recurrence within 5 years. The same conclusion was also derived from immunohistochemical staining using FFPE tissue sections from the CRC tumors in the present study. The percentage of OATP4A1-positive cells present in the tumor, immune, and stroma cells and the adjacent mucosa cells were not found to be associated with the OATP4A1 variant present in the patients.

In addition, the effect of SNPs in OATP4A1 on the functional properties of the OATP4A1 transporter was analyzed. SNPs in SLCO genes encoding for the different OATPs were described to alter the subcellular localization of the proteins, causing a shift of the transporter from the plasma membrane to the cytosol $(47,48)$. Therefore, these results suggested that SNPs in SLCO genes may also alter the transport functionality of the OATPs. For example, a nonsynonymous SNP in OATP1B1 was previously identified to change its phosphorylation status, subcellular localization, and the ability to transport its substrate estradiol glucuronide (49).

In the present study, the intracellular localization of the OATP4A1 variants and their functional properties as transporters were investigated following transfection of wtOATP4A1 and its variants in the human cancer A431 cell 
line and Sf9 insect cells. As predicted from the amino acid sequence of OATP4A1, immunoreactive bands at $\sim 70 \mathrm{kDa}$ were observed in the western blot images generated from Sf9 cell homogenates. It was also demonstrated that the two SNPs did not alter the membrane localization of the protein or its function as a transporter for the fluorescent dye sodium fluorescein. Therefore, it can be hypothesized that other OATP substrates including steroid hormones, prostaglandins and metabolic products can also be transported in an analogous manner by wt and variant OATP4A1. CRC recurrence and its outcome in patients are unlikely to be affected by these polymorphisms.

The location of the two SNPs in the N-terminal cytoplasmic domains, which is also the first intracellular loop of the protein, is distant to the region described to be important for the transport of OATP. Based on the comparative analysis of OATPs in multiple species, OATP-mediated transport is considered to occur through a central, positively charged pore, which is known as being a typical example of the rocker-switch type of transporters (4). Previous studies showed that transmembrane domain 10 is important for initial substrate recognition and its subsequent translocation across the membrane in OATP1B1 and OATP1B3 (50). Other transmembrane domains, such as the extracellular loop 6, have also been demonstrated to be important for OATP transport function, as was demonstrated in these 2 families of OATP1 proteins (51).

In conclusion, the results from the present study demonstrated that the 2 most frequent polymorphisms in the OATP4A1 gene were not associated with an increased predisposition for $\mathrm{CRC}$, nor did they alter the functional properties of the transporter, suggesting that the two OATP4A1 variants are unlikely to be risk factors for CRC development and early recurrence. In the future, larger studies investigating other variants of OATP4A1 should be conducted to facilitate the improvement in our understanding of the association between these transporters and personalized CRC risk factors.

\section{Acknowledgements}

The authors would like to thank Ms. Erika Bajna (Institute of Pathophysiology and Allergy Research, Center for Pathophysiology, Infectiology and Immunology, Medical University of Vienna, Vienna, Austria) for her technical assistance.

\section{Funding}

This study was funded by a grant from the Austrian Science Fund (FWF) awarded to WJ (grant no. I 3417-B31), the Anniversary Fund of the Oesterreichische Nationalbank (Austrian National Bank), project Nr. 17278 awarded to DM, and a grant from the Hungarian Research, Development and Innovation Office awarded to CÖ-L (grant no. OTKA FK 128751, CÖL). The work of CÖ-L was also supported by the János Bolyai Research Scholarship of the Hungarian Academy of Sciences (2015-2020).

\section{Availability of data and materials}

All data generated or analyzed during this study are included in this published article.

\section{Authors' contributions}

TT, CÖL and WJ participated in research design and supervision of the project. MS and ON conducted the experiments, and VBA and CA contributed to data analysis. AT and HA performed genetic analysis. AR and CA provided research samples and clinical data. AG, AT, DM and HA performed data analysis. VBA, TT, WJ and CÖL wrote the manuscript with substantial intellectual contributions from all authors. All authors read and approved the final manuscript.

\section{Ethics approval and consent to participate}

The present study was performed following the Declaration of Helsinki and the Ethical guidelines of the Institutions summarized in https://www.medunigraz.at/ethikkommission/index_dwnld.html. The guidelines of the Ethical Committee of the City of Vienna for retrospective studies (25) ( $\$ 15 a$ Abs. 3a des Wiener Krankenanstaltengesetzes) were applied. Informed consent was provided by the participants.

\section{Patient consent for publication}

Not applicable.

\section{Competing interests}

The authors declare that they have no competing interests.

\section{References}

1. Ferlay J, Colombet M, Soerjomataram I, Dyba T, Randi G, Bettio M, Gavin A, Visser O and Bray F: Cancer incidence and mortality patterns in Europe: Estimates for 40 countries and 25 major cancers in 2018. Eur J Cancer 103: 356-387, 2018.

2. Kleberg K, Jensen GM, Christensen DP, Lundh M, Grunnet LG, Knuhtsen S, Poulsen SS, Hansen MB and Bindslev N: Transporter function and cyclic AMP turnover in normal colonic mucosa from patients with and without colorectal neoplasia. BMC Gastroenterol 12: 78, 2012.

3. Rawłuszko-Wieczorek AA, Horst N, Horbacka K, Bandura AS, Świderska M, Krokowicz P and Jagodziński PP: Effect of DNA methylation profile on OATP3A1 and OATP4A1 transcript levels in colorectal cancer. Biomed Pharmacother 74: 233-242, 2015.

4. Stieger B and Hagenbuch B: Organic anion-transporting polypeptides. Curr Top Membr 73: 205-232, 2014.

5. Hagenbuch B and Stieger B: The SLCO (former SLC21) superfamily of transporters. Mol Aspects Med 34: 396-412, 2013.

6. Svoboda M, Riha J, Wlcek K, Jaeger W and Thalhammer T: Organic anion transporting polypeptides (OATPs): Regulation of expression and function. Curr Drug Metab 12: 139-153, 2011.

7. Obaidat A, Roth M and Hagenbuch B: The expression and function of organic anion transporting polypeptides in normal tissues and in cancer. Annu Rev Pharmacol Toxicol 52: 135-151, 2012.

8. König J: Uptake transporters of the human OATP family: Molecular characteristics, substrates, their role in drug-drug interactions, and functional consequences of polymorphisms. Handb Exp Pharmacol: 1-28, 2011 doi: 10.1007/978-3-642-14541-4_1.

9. Patik I, Kovacsics D, Német O, Gera M, Várady G, Stieger B, Hagenbuch B, Szakács G and Özvegy-Laczka C: Functional expression of the 11 human organic anion transporting polypeptides in insect cells reveals that sodium fluorescein is a general OATP substrate. Biochem Pharmacol 98: 649-658, 2015.

10. Schuster VL: Prostaglandin transport. Prostaglandins Other Lipid Mediat 68-69: 633-647, 2002.

11. Nguyen TT, Ung TT, Kim NH and Jung YD: Role of bile acids in colon carcinogenesis. World J Clin Cases 6: 577-588, 2018. 
12. Gilligan LC, Gondal A, Tang V, Hussain MT, Arvaniti A, Hewitt AM and Foster PA: Estrone sulfate transport and steroid sulfatase activity in colorectal cancer: Implications for hormone replacement therapy. Front Pharmacol 8: 103, 2017.

13. Chen C, Gong X, Yang X, Shang X, Du Q, Liao Q, Xie R, Chen Y and $\mathrm{Xu}$ J: The roles of estrogen and estrogen receptors in gastrointestinal disease. Oncol Lett 18: 5673-5680, 2019.

14. LavasaniS,ChlebowskiRT,PrenticeRL,KatoI,Wactawski-WendeJ, Johnson KC, Young A, Rodabough R, Hubbell FA, Mahinbakht A and Simon MS: Estrogen and colorectal cancer incidence and mortality. Cancer 121: 3261-3271, 2015.

15. Buxhofer-Ausch V, Secky L, Wlcek K, Svoboda M, Kounnis V, Briasoulis E, Tzakos AG, Jaeger $\mathrm{W}$ and Thalhammer $\mathrm{T}$ : Tumor-specific expression of organic anion-transporting polypeptides: Transporters as novel targets for cancer therapy. J Drug Deliv 2013: 863539, 2013

16. Buxhofer-Ausch V, Sheikh M, Ausch C, Zotter S, Bauer H, Mollik M, Reiner A, Gleiss A, Jäger W, Sebesta C, et al: Abundance of the Organic Anion-transporting Polypeptide OATP4A1 in Early-stage colorectal cancer patients: Association with disease relapse. Appl Immunohistochem Mol Morphol 27: 185-194, 2019.

17. Ban MJ, Ji SH, Lee CK, Bae SB, Kim HJ, Ahn TS, Lee MS, Baek MJ and Jeong D: Solute carrier organic anion transporter family member 4A1 (SLCO4A1) as a prognosis marker of colorectal cancer. J Cancer Res Clin Oncol 143: 1437-1447, 2017.

18. Lee HH and Ho RH: Interindividual and interethnic variability in drug disposition: Polymorphisms in organic anion transporting polypeptide 1B1 (OATP1B1; SLCO1B1). Br J Clin Pharmacol 83: $1176-1184,2017$.

19. Kalliokoski A and Niemi M: Impact of OATP transporters on pharmacokinetics. Br J Pharmacol 158: 693-705, 2009.

20. Zhang B and Lauschke VM: Genetic variability and population diversity of the human SLCO (OATP) transporter family. Pharmacol Res 139: 550-559, 2019.

21. Lee W, Belkhiri A, Lockhart AC, Merchant N, Glaeser H, Harris EI, Washington MK, Brunt EM, Zaika A, Kim RB and El-Rifai W: Overexpression of OATP1B3 confers apoptotic resistance in colon cancer. Cancer Res 68: 10315-10323, 2008.

22. Falkowski S, Woillard JB, Postil D, Tubiana-Mathieu N Terrebonne E, Pariente A, Smith D, Guimbaud R, Thalamas C, Rouguieg-Malki K, et al: Common variants in glucuronidation enzymes and membrane transporters as potential risk factors for colorectal cancer: A case control study. BMC Cancer 17: 901, 2017.

23. Evangeli L, Ioannis S, Valentinos K, Antigony M, Elli I, Eleftheria H, Vasiliki G and Evangelos B: SLCO1B3 screening in colorectal cancer patients using High-Resolution Melting Analysis method and immunohistochemistry. Tumour Biol 39: 1010428317691176, 2017

24. UICC (International Union Against Cancer). TNM Classification of Malignant Tumors. Sobin LH, Gospodarowicz MK and Wittekind CH (eds). 7th edition. Blackwell, Oxford, pp25, 2010.

25. https://www.ris.bka.gv.at/eli/lgbl/WI/1987/23/P15a/LWI40012343, Accessed: Aug 5, 2020

26. Fischer AH, Jacobson KA, Rose J and Zeller R: Hematoxylin and eosin staining of tissue and cell sections. CSH Protoc: May 1, 2008 (Epub ahead of print). doi: 10.1101/pdb.prot4986.

27. Slatkin M: Linkage disequilibrium-understanding the evolutionary past and mapping the medical future. Nat Rev Genet 9: 477-485, 2008.

28. Machiela MJ and Chanock SJ: LDlink: A web-based application for exploring population-specific haplotype structure and linking correlated alleles of possible functional variants. Bioinformatics 31: 3555-3557, 2015.

29. Rogojanu R, Thalhammer T, Thiem U, Heindl A, Mesteri I, Seewald A, Jäger W, Smochina C, Ellinger I and Bises G Quantitative image analysis of epithelial and stromal area in histological sections of colorectal cancer: An emerging diagnostic tool. BioMed Res Int 2015: 569071, 2015.

30. Haisan A, Rogojanu R, Croitoru C, Jitaru D, Tarniceriu C, Danciu $M$ and Carasevici E: Digital microscopy assessment of angiogenesis in different breast cancer compartments. BioMed Res Int 2013: 286902, 2013

31. Kolacsek O, Krízsik V, Schamberger A, Erdei Z, Apáti A Várady G, Mátés L, Izsvák Z, Ivics Z, Sarkadi B and Orbán TI: Reliable transgene-independent method for determining sleeping beauty transposon copy numbers. Mob DNA 2: 5, 2011

32. Gál C, Hegedüs C, Szakács G, Váradi A, Sarkadi B and Özvegy-Laczka C: Mutations of the central tyrosines of putative cholesterol recognition amino acid consensus (CRAC) sequences modify folding, activity, and sterol-sensing of the human ABCG2 multidrug transporter. Biochim Biophys Acta 1848: 477-487, 2015.
33. Ozvegy C, Váradi A and Sarkadi B: Characterization of drug transport, ATP hydrolysis, and nucleotide trapping by the human ABCG2 multidrug transporter. Modulation of substrate specificity by a point mutation. J Biol Chem 277: 47980-47990, 2002.

34. Tschantz WR, Pfeifer ND, Meade CL, Wang L, Lanzetti A, Kamath AV, Berlioz-Seux F and Hashim MF: Expression, purification and characterization of the human membrane transporter protein OATP2B1 from Sf9 insect cells. Protein Expr Purif 57: 163-171, 2008.

35. Patik I, Székely V, Német O, Szepesi Á, Kucsma N, Várady G, Szakács G, Bakos É and Özvegy-Laczka C: Identification of novel cell-impermeant fluorescent substrates for testing the function and drug interaction of Organic Anion-transporting polypeptides, OATP1B1/1B3 and 2B1. Sci Rep 8: 2630, 2018.

36. Tusnády GE and Simon I: The HMMTOP transmembrane topology prediction server. Bioinformatics 17: 849-850, 2001

37. Hagenbuch B and Meier PJ: The superfamily of organic anion transporting polypeptides. Biochim Biophys Acta 1609: 1-18, 2003.

38. Thakkar N, Lockhart AC and Lee W: Role of organic anion-transporting polypeptides (OATPs) in cancer therapy. AAPS J 17: 535-545, 2015.

39. Kovacsics D, Patik I and Özvegy-Laczka C: The role of organic anion transporting polypeptides in drug absorption, distribution, excretion and drug-drug interactions. Expert Opin Drug Metab Toxicol 13: 409-424, 2017.

40. Sissung TM, Goey AK, Ley AM, Strope JD and Figg WD: Pharmacogenetics of membrane transporters: A review of current approaches. Methods Mol Biol 1175: 91-120, 2014.

41. Teft WA, Welch S, Lenehan J, Parfitt J, Choi YH, Winquist E and Kim RB: OATP1B1 and tumour OATP1B3 modulate exposure, toxicity, and survival after Irinotecan-based chemotherapy. $\mathrm{Br}$ J Cancer 112: 857-865, 2015.

42. Rhodes KE, Zhang W, Yang D, Press OA, Gordon M, Vallböhmer D, Schultheis AM, Lurje G, Ladner RD, Fazzone W, et al: ABCB1, SLCO1B1 and UGT1A1 gene polymorphisms are associated with toxicity in metastatic colorectal cancer patients treated with first-line irinotecan. Drug Metab Lett 1: 23-30, 2007.

43. Roth M, Obaidat A and Hagenbuch B: OATPs, OATs and OCTs: The organic anion and cation transporters of the SLCO and SLC22A gene superfamilies. Br J Pharmacol 165: 1260-1287, 2012.

44. Aslibekyan S, Brown EE, Reynolds RJ, Redden DT, Morgan S, Baggott JE, Sha J, Moreland LW, O'Dell JR, Curtis JR, et al: Genetic variants associated with methotrexate efficacy and toxicity in early rheumatoid arthritis: Results from the treatment of early aggressive rheumatoid arthritis trial. Pharmacogenomics J 14: 48-53, 2014.

45. Eektimmerman F, Swen JJ, Böhringer S, Aslibekyan S, Allaart CF and Guchelaar HJ: SLC04A1, SLC22A2 and SLC28A2 variants not related to methotrexate efficacy or toxicity in rheumatoid arthritis patients. Pharmacogenomics 19: 613-619, 2018.

46. Fairley S, Lowy-Gallego E, Perry E and Flicek P: The International Genome sample resource (IGSR) collection of open human genomic variation resources. Nucleic Acids Res 48: D941-D947, 2020.

47. Durmus S, Lozano-Mena G, van Esch A, Wagenaar E, van Tellingen $O$ and Schinkel AH: Preclinical mouse models to study human OATP1B1- and OATP1B3-mediated drug-drug interactions in vivo. Mol Pharm 12: 4259-4269, 2015.

48. Alam K, Crowe A, Wang X, Zhang P, Ding K, Li L and Yue W: Regulation of organic anion transporting polypeptides (OATP) 1B1- and OATP1B3-mediated transport: An updated review in the context of OATP-mediated drug-drug interactions. Int J Mol Sci 19: E855, 2018.

49. Crowe A, Zheng W, Miller J, Pahwa S, Alam K, Fung KM, Rubin E, Yin F, Ding K and Yue W: Characterization of plasma membrane localization and phosphorylation status of organic anion transporting polypeptide (OATP) 1B1 c.521 T $>$ C nonsynonymous single-nucleotide polymorphism. Pharm Res 36: 101, 2019.

50. Gui C and Hagenbuch B: Amino acid residues in transmembrane domain 10 of organic anion transporting polypeptide 1B3 are critical for cholecystokinin octapeptide transport. Biochemistry 47: 9090-9097, 2008.

51. Meier-Abt F, Mokrab Y and Mizuguchi K: Organic anion transporting polypeptides of the OATP/SLCO superfamily: Identification of new members in nonmammalian species, comparative modeling and a potential transport mode. J Membr Biol 208: 213-227, 2005

This work is licensed under a Creative Commons Attribution-NonCommercial-NoDerivatives 4.0 International (CC BY-NC-ND 4.0) License. 